\title{
La connectivité sédimentaire dans la haute chaîne pyrénéenne par l'analyse de la crue de juin 2013 : le rôle des formations superficielles
}

\section{Sediment connectivity in the high Pyrenees mountain range by 2013 flood analysis: role of surficial sediment storages}

\author{
Johann Blanpied ${ }^{*}$, Jean-Michel Carozza ${ }^{\mathrm{b}}$, Jean-Marc Antoine ${ }^{\text {a }}$ \\ ${ }^{a}$ GEODE UMR 5602 CNRS, Université Jean Jaurès - 5 allée Antonio Machado, 31058 Toulouse, France. \\ ${ }^{\mathrm{b}}$ LIENSS UMR 7266 CNRS, Université de La Rochelle - 1 Parvis Fernand Braudel, 17042 La Rochelle, France.
}

INFORMATION SUR LARTICLE

Article soumis le 4 mai 2018

Reçu sous sa forme révisée le 10 octobre 2018

Définitivement accepté le 22 décembre 2018.

*Auteur correspondant. Tél : +33 (0)561503626; Courriels

johann.blanpied@univ-tlse2.fr (J. Blanpied) jean-michel.carozza@univ-lr.fr (J.-M. Carozza) antoine@univ-tlse2.fr (J.-M. Antoine)

\begin{abstract}
RÉSUMÉ
L' (in)efficacité du couplage versant/lit est particulièrement mise en lumière lors dévènements hydro-climatiques extrêmes. La crue majeure de juin 2013 dans les Pyrénées centrales a montré le rôle des formations superficielles dans la constitution de la charge solide des écoulements. En zone de montagne, les paramètres morphométriques sont également une variable de contrôle de la connectivité sédimentaire. Dans cette étude, la dynamique sédimentaire de la crue est étudiée sur les quatre bassins versants qui ont été les plus marqués par la crue. L’analyse se fonde sur le calcul d’un indice morphométrique de connectivité sédimentaire réalisé à partir d'un MNT, qui est ensuite croisé avec la cartographie des formations superficielles et des zones actives lors de la crue. Cette méthodologie permet de comparer la connectivité théorique/structurelle des sous-bassins versants et formations superficielles à la connectivité réelle/fonctionnelle lors de la crue. À la différence des grandes crues historiques passées, cette comparaison fait ressortir la faible contribution des bassins tributaires de petite taille qui possèdent pourtant une connectivité sédimentaire théorique importante. Les têtes de bassins et les dépôts du domaine glaciaire et périglaciaire nont pas fourni de matériaux lors de la crue et sont des zones déconnectées. A contrario, les dépôts post-glaciaires adjacents au réseau hydrographique du fond de vallée sont les plus connectés et les plus contributifs. En effet, la connectivité sédimentaire latérale se réalise principalement entre les formations superficielles de bas de versant de moyenne et basse vallée, adjacents au collecteur principal. Notre étude souligne le potentiel de l'indice morphométrique appuyé par la cartographie géomorphologique pour caractériser la connectivité sédimentaire de la haute chaîne pyrénéenne en contexte d’événement majeur.
\end{abstract}

Mots clés : Pyrénées centrales, connectivité sédimentaire, modélisation, formations superficielles, crue centennale.

\begin{abstract}
The (in)efficiency of sediment connectivity between hillslopes and river channel is highlighted during extreme hydroclimatic events. The high-magnitude flood of June 2013 in the Central Pyrenees has showed the role of inherited surficial sediment storages in the feeding of river sediment load. In mountain areas, morphometric parameters are also a controlling factor of the sediment connectivity. In this study, sediment routing during the flood studied in the four most affected catchments. The analysis is based on a GIS morphometric index of sediment connectivity derived from 5-m DTM. It is combined with classical geomorphic mapping of surficial sediment storages and active areas during the flood. This methodology allowed to compare the structural connectivity of sub-catchments and surficial sediment storages with functional connectivity during the flood. Compare to the historical past major floods, it displayed the low sediment supply of small tributaries with high average connectivity. Headwater areas and glacial sediment storages have not supplied sediments during the flood and acted as disconnected areas according to the index. Conversely, post glacial sediment storages in the valley bottom were the most connected and contributive stores. Lateral sediment connectivity occurred mainly between storages of the mid and low part of the valley which are adjacent to the main river. Our study highlights the potential of the morphometric index supported by geomorphological mapping to characterize the central Pyrenean connectivity in a context of a low frequency high magnitude events.
\end{abstract}

Keywords: Central Pyrenees, sediment connectivity, modelling, surficial sediment storages, 100-year flood.

\section{Introduction}

Les évènements majeurs, ie., les crues de basse fréquence et haute magnitude (BFHM), comme la crue de juin 2013 dans les Pyrénées centrales, sont des épisodes d'activation de la connectivité sédimentaire au réseau hydrographique. La connaissance du fonctionnement des apports sédimentaires au chenal (activité des versants, érosions de berges, contribution des tributaires latéraux, etc.) est essentielle pour la mise en place d'une gestion concertée des risques torrentiels (Eaton et al., 2003 ; Hungr et al., 2005), pour une gestion durable de la charge sédimentaireet pour la compréhension de l'évolution paysagère (Savi et al., 2013). Initialement conceptualisée à une échelle locale à travers le 
couplage versant/lit (Harvey, 1991, 2001), la notion de connectivité sédimentaire a été ensuite et est définie à présent comme le degré d' (in)efficacité (Harvey, 2002) du couplage entre les sources sédimentaires ou le système de versants (Brierley et al., 2006) et le chenal (Hooke, 2003). Actuellement, le terme de couplage est utilisé pour l'étude des liens unitaires entre les unités géomorphologiques à l'échelle locale alors que le terme de connectivité recouvre l'ensemble de ces liens à l'échelle du bassin versant. La connectivité sédimentaire est mobilisée pour l'étude de bilan sédimentaire (Warburton, 2010), pour expliquer les changements morphologiques du chenal (Chiverell et al., 2009; Harvey, 2012), pour comprendre les changements paysagers sur le temps long (occupation du sol, climat, tectonique), ainsi qu'en biogéomorphologie et en écologie (Sandercock et Hooke, 2011). Ce concept est devenu multidimensionnel (Fryirs et al., 2007 ; Fryirs, 2013) englobant: (i) la connectivité verticale c'est-à-dire les échanges sédimentaires et géochimiques entre le lit et la subsurface; (ii) la connectivité longitudinale, de la source sédimentaire à la zone de stockage ; (iii) la connectivité latérale, c'est-à-dire les apports latéraux des tributaires et les apports en masse, les glissements de terrain et des berges. Lorsque certains stocks sédimentaires jouent le rôle de barrière au transit sédimentaire (Fryirs, 2013), le terme de "disconnectivity " est employé et traduit l'interruption de la cascade sédimentaire latérale ou longitudinale. À l'échelle $\mathrm{du}$ bassin versant, cela peut être une déconnexion entre stocks sédimentaires du versant et le réseau hydrographique (Heckmann et Schwanghart, 2013). Différents facteurs de contrôle influencent et caractérisent l' (in)efficacité spatiale et temporelle de la connectivité sédimentaire, notamment la capacité de transport et la géométrie du réseau hydrographique (Rice, 2017 ; Gran et Czuba, 2017), mais également la taille et la forme des bassins versants et des fonds de vallées (Fryirs et Brierley, 2001 ; Cossart, 2016). La connectivité sédimentaire peut également être abordée en distinguant la connectivité théorique structurelle de la connectivité réelle fonctionnelle. Cette terminologie issue de l'étude de la connectivité des écosystèmes (Merriam, 1984), a été étendue à la géomorphologie où la connectivité structurelle s'apparente à l'agencement des liens à l'échelle du bassin versant alors que la connectivité fonctionnelle s'intéresse aux interactions qui existent plus localement et temporairement dans le bassin versant (Cossart et al., 2017). Depuis les premières études sur le couplage géomorphologique et la connectivité (Caine et Swanson, 1989), les approfondissements de la notion de connectivité sédimentaire ont porté sur la formalisation théorique et la modélisation notamment au travers des Systèmes d'Information Géographique (Hooke, 2003 ; Croke et al., 2005) qui ont permis un changement d'échelle, vers l'étude de territoires plus étendus. Les analyses réseaux et la théorie des graphes ont été utilisées pour l'étude de l'organisation de la cascade sédimentaire en prenant en compte les stocks sédimentaires et les processus (Heckmann et Schwanghart, 2013 ; Heckmann et al., 2015 ; Cossart et Fressard, 2017). D'autres approches se sont focalisées sur le calcul d'un indice géomorphométrique de connectivité sédimentaire (Borselli et al., 2008 ; Cavalli et al., 2013), basé sur le traitement d'un Modèle Numérique de Terrain. Cette approche a été validée et son potentiel pour l'estimation de la connectivité sédimentaire à différentes échelles est aujourd'hui reconnu (Gay et al., 2015 ; LopezVincente et al., 2016 ; Nicol et Brierley, 2016 ; Surian et al., 2016 ; Tiranti et al., 2016), particulièrement en zone de montagne où la topographie est le facteur de contrôle majeur de l'acheminement de sédiments au réseau hydrographique. Cet indice a été utilisé pour l'étude de la cascade sédimentaire d'un bassin versant suisse à partir du croisement de l'indice de connectivité avec des topo- séquences et des stocks sédimentaires (Messenzehl et al., 2014). La cascade sédimentaire représente l'emboîtement hiérarchique d'unités de stockage sédimentaire (Chorley et al., 1984 ; Evans, 2012). Largement étudiée dans différentes parties des Alpes, elle permet une meilleure évaluation du couplage versant/lit par l'étude des dynamiques des transferts sédimentaires (Theler et al., 2010). L'indice de connectivité a également été utilisé pour effectuer une analyse diachronique de la connectivité des sources sédimentaires dans les Alpes italiennes (Cavalli et al., 2016). Il n'a cependant encore jamais été exploité pour la compréhension du fonctionnement d'un évènement hydrogéomorphologique majeur. Ces évènements jouent un rôle particulier dans le fonctionnement du système torrentiel car ils favorisent a priori une augmentation exceptionnelle de la connectivité sédimentaire mais qui reste éphémère. En effet, au cours de ce type d'évènements, les «buffers, barriers et blankets» de Fryirs et al. (2007) qui entravent le processus d'apport sédimentaire sont réactivés et augmentent l'efficacité de la connectivité sédimentaire. Ces événements sont susceptibles de produire des dynamiques particulières et très morphogènes qui doivent être étudiées de manière spécifique.

La crue majeure du 18-19 juin 2013 dans les Pyrénées centrales a remobilisé des formations superficielles de fond de vallée et donné lieu à des élargissements importants de la bande active sur certains torrents et rivières torrentielles. Dans ce travail, nous cherchons à mettre en évidence les spécificités, sous l'angle de la connectivité des sources sédimentaires, de cet évènement par rapport aux grandes crues historiques passées comparables à celle de juin 2013 (1897, 1937, etc.). Les premières observations de terrain nous ont amené à formuler l'hypothèse d'une faible contribution des têtes de bassins versants lors de la crue de juin 2013, contrairement à ce que semblent indiquer les données disponibles pour les épisodes antérieurs. Ce travail remobilise la méthode proposée par Cavalli et al. (2013) et Messenzehl et al. (2014). Dans cette perspective, les objectifs de l'étude sont (i) d'évaluer le rôle des formations superficielles dans la constitution de la charge solide du chenal lors de la crue puis (ii) d'identifier les variables qui régissent la connectivité sédimentaire de ces bassins versants.

\section{Contexte et site d'étude}

Les Pyrénées centrales correspondent à la partie des Pyrénées la plus élevée abritant les plus hauts sommets et les derniers glaciers pyrénéens. Elles s'étendent d'est en ouest sur les départements de l'Ariège, de la Haute-Garonne et des Hautes-Pyrénées sur environ $200 \mathrm{~km}$.

L'étude porte sur les quatre bassins versants qui ont été les plus affectés par la crue de juin 2013 à savoir le bassin du Bastan, de la Neste de Badet, de la Neste d'Oô et du Lys (fig. 1). Le Bastan est un affluent du Gave de Pau dans le bassin de l'Adour. Les trois autres torrents sont des sous-affluents de la Garonne par l'intermédiaire de la Neste et de la Pique. Ces rivières, d'ordre 4 selon la classification de Strahler, présentent un régime nivo-pluvial. Les altitudes de leurs bassins versants s'échelonnent entre $658 \mathrm{~m}$ et $3219 \mathrm{~m}$ et leur superficie est comprise entre $21 \mathrm{~km}^{2}$ et $101 \mathrm{~km}^{2}$ (tab. 1). Les Pyrénées centrales se caractérisent par un climat sub-océanique exposé aux perturbations d'ouest-nord-ouest pour les vallées de la Garonne et les environs du Pic du Midi de Bigorre. Sur la haute chaîne frontalière, le climat est également sub-océanique à tendance plus froide mais soumis aux perturbations d'ouest à sud-ouest (Vigneau, 1999).

Cette dernière configuration est responsable de la crue de juin 2013 lorsque le flux de sud-ouest a débordé sur les versants français abondamment enneigés, accompagné d'intenses précipitations en 


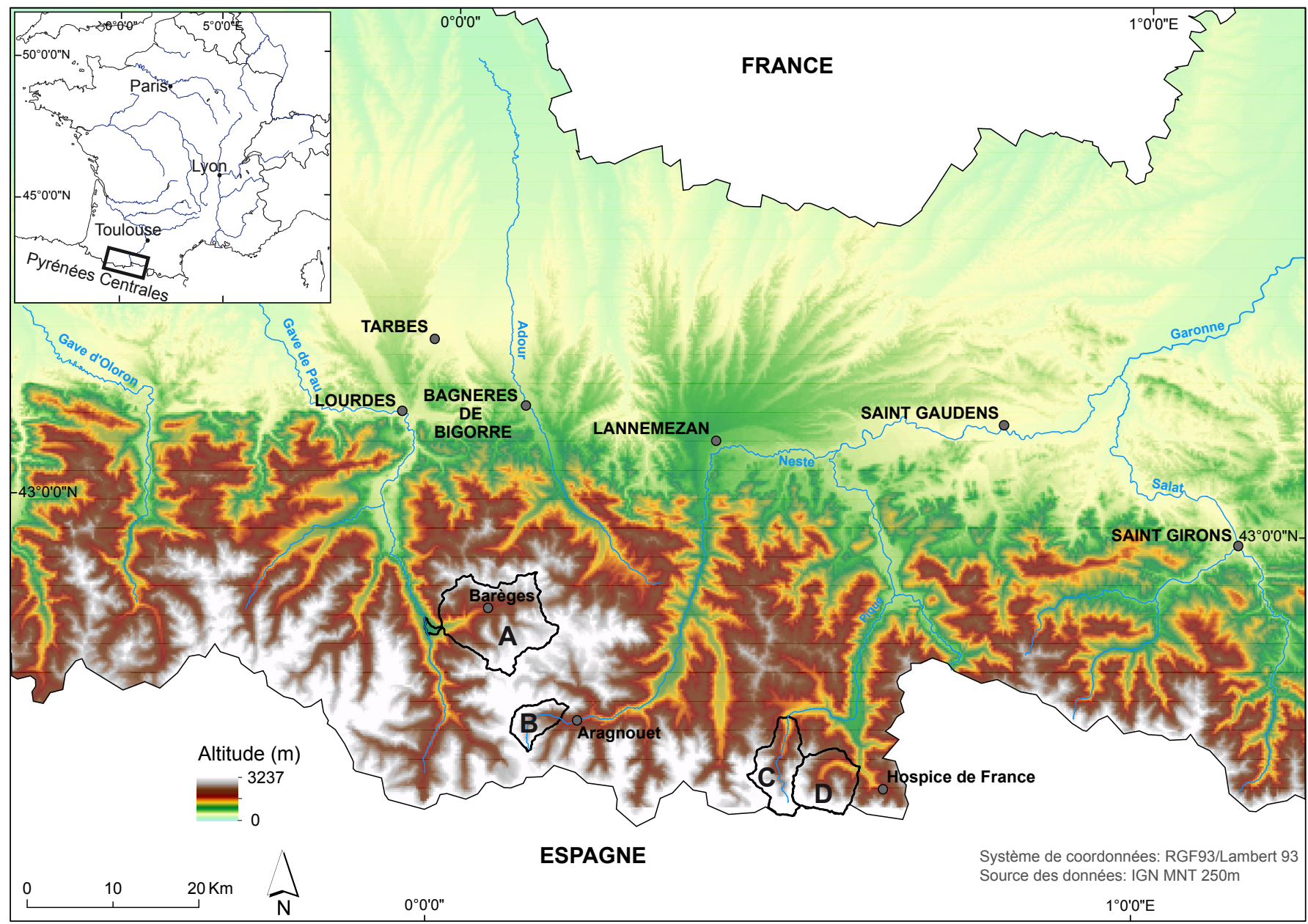

Fig. 1 - Carte de localisation des sites d'étude.

1. Zone d'étude ; 2. Ville principale ; 3. Cours d'eau principal; A : Bassin du Bastan ; B : Bassin de la Neste de Badet ; C : Bassin de la Neste d'Oô ; D : Bassin du Lys.
Fig. 1 - Location map of study sites.

1. Catchment study; 2. Major city; 3. Main river; A: Bastan catchment; B: Neste de Badet catchment; C: Neste d'Oô catchment; D: Lys catchment.
Tab. 1 - Caractéristiques morphométriques principales des bassins étudiés.

Tab.1 - Main morphometric characteristics of studies sites.

\begin{tabular}{|c|c|c|c|c|c|c|c|c|c|}
\hline Site d'étude & $\begin{array}{c}\text { Surface } \\
\left(\mathrm{km}^{2}\right)\end{array}$ & $\begin{array}{c}\text { Altitude } \\
\text { minimale- } \\
\text { maximale }(\mathrm{m})\end{array}$ & $\begin{array}{l}\text { Pente } \\
\text { moyenne } \\
\left(^{\circ}\right)\end{array}$ & $\begin{array}{l}\text { Indice de } \\
\text { compacité } \\
\text { de Gravelius }\end{array}$ & $\begin{array}{l}\text { Indice } \\
\text { de } \\
\text { Melton }\end{array}$ & $\begin{array}{l}\text { Densité de } \\
\text { drainage } \\
\left(\mathrm{km} / \mathrm{km}^{2}\right)\end{array}$ & $\begin{array}{l}\text { Précipitations } \\
\text { moyennes } \\
(\mathrm{mm})\end{array}$ & $\begin{array}{l}\text { Augmentation de la } \\
\text { largeur moyenne de } \\
\text { la bande active (\%) } \\
\text { post crue juin } 2013\end{array}$ & $\begin{array}{c}\text { Surface des } \\
\text { formations superfi- } \\
\text { cielles en \% de la } \\
\text { surface du bassin }\end{array}$ \\
\hline Bastan & 101 & $658-3091$ & 29 & 1,36 & 0,14 & 1,27 & 1250 & 524 & 67 \\
\hline Neste de Badet & 21 & $1408-3159$ & 32 & 1,27 & 0,3 & 1,81 & 1066 & 109 & 54 \\
\hline Neste d'Oô & 43 & $964-3219$ & 32 & 1,42 & 0,32 & 1,64 & 1633 & 356 & 45 \\
\hline Lys & 44 & $846-3116$ & 32 & 1,13 & 0,34 & 1,9 & 1633 & 172 & 42 \\
\hline
\end{tabular}

Réalisé à partir de la RGE Alti 5 m, de la BD Carthage ${ }^{\circledR} \mathrm{IGN}$.

Based on ${ }^{\circledR}$ GN RGE Alti 5 m and Carthage Database).

haute montagne. Le total des précipitations du 18 et 19 juin 2013 sur les stations les plus proches des zones d'étude est supérieur à $100 \mathrm{~mm}$ (Barèges, $103 \mathrm{~mm}$; Aragnouet, $127 \mathrm{~mm}$; Hospice de France, $133 \mathrm{~mm}$ ) et ces cumuls ont encore été plus importants en altitude sur les crêtes frontalières. De plus, la contribution de la fonte nivale au pic de crue est estimée à environ $40 \%$ (CHE, 2014 ; Pineda et al., 2013). Cette crue pluvio-nivale est de l'ordre de la crue centennale pour les quatre bassins étudiés (ETRM, 2014 ; IGA et al., 2014). L'élargissement des bandes actives de plus de $100 \mathrm{~m}$ à certains endroits a engendré des dégâts importants sur les infrastructures humaines (réseau routier et habitations), des érosions de parcelles en fond de vallée et une remobilisation des formations superficielles.
Les quatre bassins s'inscrivent exclusivement dans la zone axiale pyrénéenne, communément appelée " haute chaîne primaire ". Dominés par les massifs granitiques de Cauterets, du Néouvielle et du Crabioules, les versants de hautes et moyennes altitudes sont constitués par les séries métamorphiques schisto-cristallines et schisto-calcaires principalement datées du Carbonifère ou par les calcaires du Dévonien au contact des séries détritiques des versants moins élevés. Ce contexte géologique se traduit par deux types de morphologie de vallée. D’une part, il s'agit de petits bassins aux versants raides directement connectés au collecteur principal étroit de fond de vallée ; ce sont des sites à forte susceptibilité avalancheuse, potentiellement propices à une connectivité sédimentaire efficace ; c'est le cas des bassins de la 
Neste de Badet et de la soulane du Bastan où les séries détritiques du Dévonien et du Carbonifère (calcaire, grès, argiles schisteuses) plus ou moins schisteuses dominent les formations superficielles de versant et de fond de vallée. D'autre part, le bassin de la Neste d'Oô, le bassin du Lys ainsi que les sous-bassins de l'ombrée du Bastan sont des vallées glaciaires typiques, dominées par de larges cirques glaciaires accueillant de nombreux lacs d'ombilics au sein du domaine glaciaire et périglaciaire granitique. Ces zones de surcreusement glaciaire débouchent par l'intermédiaire de gorges sur des auges glaciaires aux versants latéraux abrupts. Dans le fond de ces auges, la bande active s'élargit préférentiellement lors des épisodes de crue.

Outre les données géologiques, les formations superficielles revêtent une importance majeure dans la fourniture sédimentaire, compte tenu de leur extension (entre $42 \%$ et $67 \%$ de la superficie du bassin) (fig. 2). En altitude, les versants sont couverts de formations glaciaires de type moraine latérale mais également de formations post-glaciaires issues de la fragmentation mécanique et de l'altération des parois. Les fonds de vallée peuvent être occupés par des accumulations glaciaires (moraines de fond, dépôts fluvio-glaciaires, dépôts glacio-lacustres, etc.) et par des formations post-glaciaires (cônes de déjection, formations alluviales). Ces fonds de vallée et pieds de versant sont des zones privilégiées pour les communications et l'installation des activités humaines où l'aménagement du territoire doit composer avec la gestion des risques et les règles d'urbanisation. Depuis la fin du XIX ${ }^{\mathrm{e}}$ siècle, voire dès le début du $\mathrm{XIX}^{\mathrm{e}}$ siècle à Barèges, des politiques de protection et de correction torrentielle et avalancheuse sur les versants ont été réalisées par le service de Restauration des Terrains en Montagne (RTM). Sous forme de reboisements et de rectification du lit de nombreuses ravines très actives (Campagne, 1902), ces corrections ont été réalisées à la suite de la période de crues fréquentes et intenses de la fin du XIX ${ }^{\mathrm{e}}$ siècle et du début $\mathrm{XX}^{\mathrm{e}}$ siècle. Outre une activité touristique très développée, avec la présence de stations de ski comme Barèges-Grand Tourmalet (Bastan) ou Piau-Engaly (Neste de Badet), ces vallées accueillent de nombreuses infrastructures hydroélectriques telles des lacs de barrage, des dérivations hydrauliques et des stations de pompage qui contribuent à la complexité du fonctionnement hydrologique des bassins.
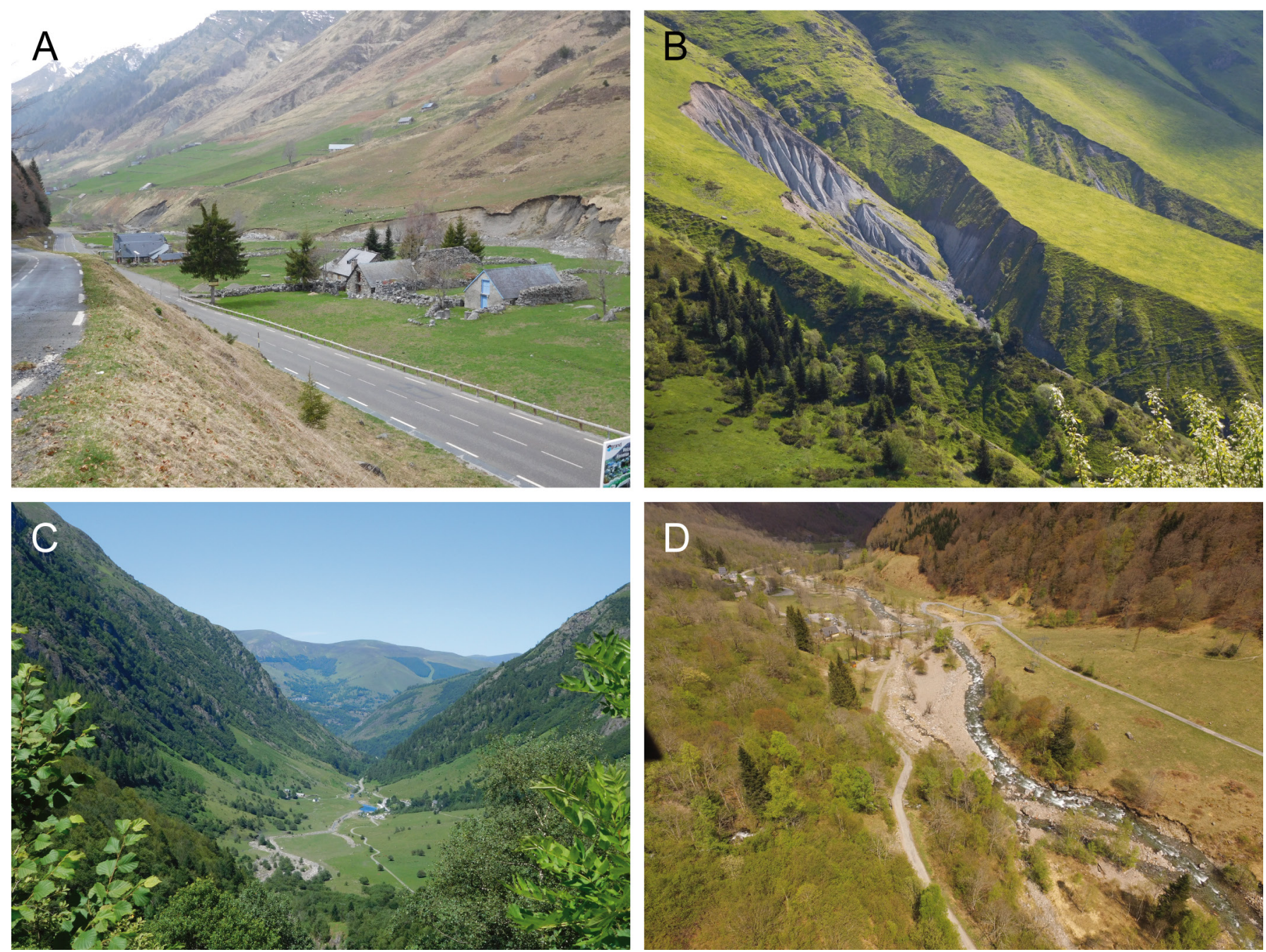

Fig. 2 - Les formations superficielles de bas de versant et de fond de vallée des quatre bassins versants.

A : Dépôts fluvio-torrentiels de fond de vallée et dépôts glaciaires hérités en rive droite sur le bassin du Gave du Bastan ; B : Ravines et dépôts glaciaires hérités dans le bassin de la Neste de Badet ; C : Cônes de déjection et dépôts fluvio-torrentiels dans la vallée en auge de la Neste d'Oô ; D : Dépôts fluvio-torrentiels et cône de déjection torrentiels en rive droite du Lys.

Fig. 2 - Surficial sediment storages on downslope part and in the valley bottom of the 4 catchments.

A: Alluvium deposits of the valley bottom and inherited glacial deposits on the right bank in Bastan catchment; B: Gullies and inherited glacial deposits in the Neste de Badet catchment; C: Debris cones and alluvium deposits in the hunging valley of the Neste d'Oô; D: Alluvial deposits and debris cones on the right bank of the Lys catchment. 


\section{Méthodologie}

\subsection{Cartographie géomorphologique}

Dans le but de caractériser la connectivité structurelle pyrénéenne, un inventaire des formations superficielles a été réalisé par photo-interprétation diachronique $\left(\mathrm{BD} \mathrm{Ortho}^{\odot}\right.$ de 2013, 2010, 2001), utilisation du MNT 5 m (RGE Alti ${ }^{\oplus}$ IGN 2013), traitements raster associés (ombrage et pente), usage de la couche SIG géologie $\left(\mathrm{BRGM}^{\odot}\right)$ et observations de terrain. Une formation superficielle se définit comme une formation continentale ou littorale élaborée à l'interface de la lithosphère, l'hydrosphère, la biosphère et l'atmosphère (Dewolf et Bourrié, 2008). Dans notre étude, ce sont des formations dites sub-autochtones et allochtones, c'est-à-dire des formations qui ont subi des déplacements plus ou moins longs dus à l'intervention de la gravité, du ruissellement et des mouvements de masse sous l'impact de la dynamique glaciaire et fluviatile. Ces formations du dernier maximum glaciaire et de la période post-glaciaire sont issues des morpho-domaines (Theler et al., 2010) fluvio-torrentiels, glacio-nivaux et gravitaires. Elles sont classées en 9 types (fig. 2-3) : les dépôts fluvio-torrentiels, les cônes de déjection torrentiels, les cônes de déjection mixtes (avalancheux et torrentiels), les éboulis, les moraines dénudées, les dépôts glaciaires hérités du dernier maximum glaciaire, les talus d'éboulis/dépôts glaciaires indifférenciés (i.e., lorsque les moraines latérales sont recouvertes d'un tablier d'éboulis) et les glissements de terrains. Un autre type a été ajouté, bien qu'il ne s'agisse pas de formation superficielle stricto sensu mais d'un processus facilement délimitable et historiquement actif lors de crues majeures et qui contribue à la fourniture sédimentaire : les ravines (fig. 2). Les sources sédimentaires structurales à savoir les parois rocheuses et les zones d'affleurement de la roche en place sur les versants ne sont pas prises en compte dans l'étude. En effet, dans les massifs granitiques peu fracturés, l'érosion mécanique et l'altération sont considérées comme faibles en l'absence notable d'altérites. De plus, les observations de terrain et la photo-interprétation diachronique
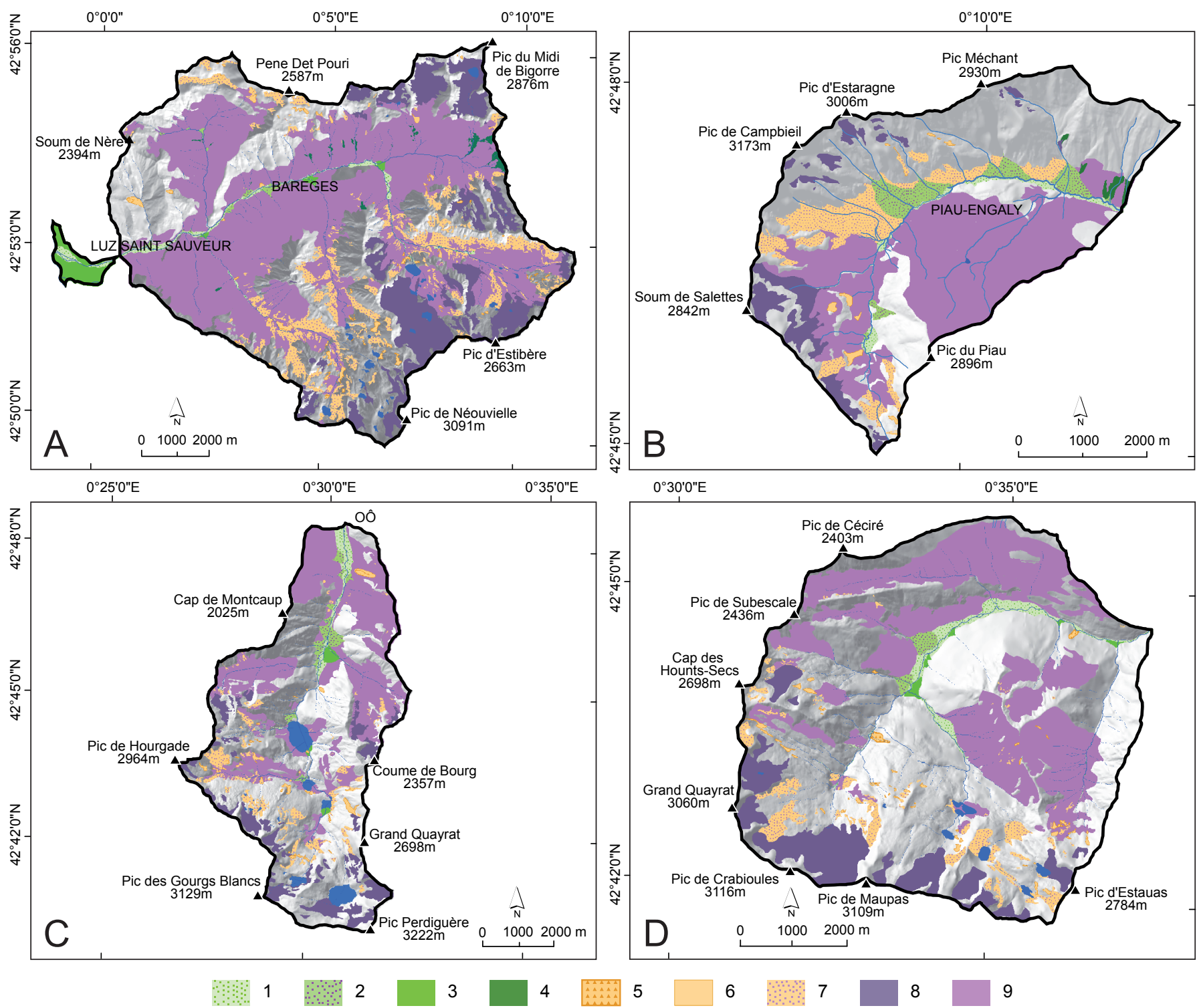

\section{5}

\section{6}

Fig. 3 - Geomorphic map of surficial geology.

Fig. 3 - Cartographie géomorphologique des formations superficielles. 1. Dépôts fluvio-torrentiels ; 2. Cônes de déjection mixtes ; 3. Cônes de déjection torrentiels ; 4. Ravines ; 5 . Glissements de terrain ; 6. Éboulis ; 7. Dépôts glaciaires/ Talus d'éboulis indifférenciés; 8 . Moraines; 9 . Dépôts glaciaires hérités. A : Bassin du Bastan ; B : Bassin de la Neste de Badet ; C : Bassin de la Neste d'Oô ; D : Bassin du Lys.

1. Alluvial deposits; 2. Mixed debris cones; 3. Debris cones; 4. Gullies; 5. Landslides; 6. Talus slope; 7. Undistinguished glacial deposits/talus slope; 8. Moraine deposits, 9. Inherited glacial deposits. A: Bastan basin; B: Neste de Badet basin; C: Neste d'Oô basin; D: Lys basin. 
ne montrent pas de grandes zones de production, de glissements de terrain ou de niches d'arrachement au sein de ces parois. La végétation qui recouvre les formations superficielles ainsi que des caractéristiques morphométriques comme la pente ou la distance au réseau hydrographique sont également prises en compte.

\subsection{Délimitation des sous-systèmes et des zones actives lors de la crue de juin 2013}

L'étude a porté sur 47 sous-bassins délimités à l'aide d'un SIG en utilisant l'outil ArcHydro avec un seuil minimal de $0,2 \mathrm{~km}^{2}$. Ils confluent avec le collecteur principal du bassin, à savoir le Lys, la Neste d'Oô, la Neste de Badet ou le Bastan. Ils sont dominés par des dynamiques soit avalancheuse, soit torrentielle. L'inventaire des données historiques de la base de données RTM et les observations de la crue de juin 2013 ont été utilisés pour déterminer le processus dominant de chaque sous-système. La dynamique renseignée est la dynamique actuelle ou sub-actuelle qui a pu évoluer notamment au gré des interventions RTM depuis plus d'un siècle. Pour analyser le rôle de la forme des bassins sur la connectivité sédimentaire, l'indice morphométrique de compacité de Gravelius a également été calculé pour chaque bassin.

La crue majeure de juin 2013 a été exceptionnelle en terme de transport sédimentaire pour ces quatre bassins versant par rapport aux Pyrénées centrales et à la haute chaîne pyrénéenne en général. Elle a mis en exergue à la fois le couplage de certaines formations superficielles avec le réseau hydrographique mais également le découplage de certaines parties de ces bassins versants, ce qui permet d'étudier la connectivité sédimentaire réelle fonctionnelle à l'échelle de l'évènement. Sur les quatre bassins, la cartographie des zones actives a été réalisée. Une zone active a été définie comme une formation sédimentaire qui a contribué de manière efficace à la formation de la charge véhiculée par le réseau hydrographique et qui présente une surface de sol nue de plus de $50 \%$ (Bravard et Petit, 2000 ; Liébault, 2003). La carte est réalisée avec le logiciel ArcGIS par photo-interprétation en comparant la BD Ortho ${ }^{\circ}$ IGN de 2010, 2013 et les photographies aériennes post-crue réalisées par le service de Restauration des Terrains en Montagne. Différentes caractéristiques ont été renseignées pour chaque zone active comme le type de formation superficielle, la localisation dans les sous-systèmes, la surface $\left(\mathrm{m}^{2}\right)$, le type de couvert végétal, la pente et la distance minimale au réseau hydrographique principal.

\subsection{Indice morphométrique de connectivité sédimentaire}

La modélisation de la connectivité sédimentaire a été réalisée à partir de l'indice de connectivité (IC) en suivant l'approche de Cavalli et al. (2013) (fig. 4). Créé à l'origine par Borselli et al. (2008), il a été développé pour l'étude de la connectivité sédimentaire en montagne par Cavalli et al. (2013). Il est issu d'un algorithme morphométrique qui permet de modéliser la connectivité potentielle entre les versants et une zone de stockage cible (réseau hydrographique, lac, barrage, réseau routier, obstacle à l'écoulement ou exutoire, etc.) à partir d'un modèle numérique de terrain :

$$
I C=\log _{10}\left(\frac{D_{u p}}{D_{d n}}\right)
$$

où $D_{u p}$ et $D_{d n}$ représentent les composants amont et aval de la connectivité. La composante amont est définie comme le potentiel de transfert vers l'aval d'une particule amont et est obtenu de la façon suivante:

$$
D_{u p}=\overline{W S} \sqrt{A}
$$

où $W$ est le facteur de pondération à partir de l'indice standardisé de rugosité (Cavalli et al., 2013 ; Trevisani et Cavalli, 2016). Il s'agit de la déviation standard de la différence entre une surface lisse et rugueuse, c'est-à-dire un résidu topographique. Il permet de considérer les obstructions locales au transfert sédimentaire. $S$ représente la pente moyenne de la zone contributive amont $(\mathrm{m} / \mathrm{m})$ et $A$ l'aire de la zone contributive amont $\left(\mathrm{m}^{2}\right)$.

Le composant aval représente la longueur du linéaire qui sépare la particule de la zone de stockage cible la plus proche. Elle est calculée ainsi :

$$
D_{d n}=\sum_{i} \frac{d_{i}}{W_{i} S_{i}}
$$

où $d$ est la longueur du linéaire le plus pentu entre la particule $i$ et la zone de stockage cible $(\mathrm{m})$. $W$ est l'indice de rugosité (a-dimensionnel) et $S$ la pente moyenne $(\mathrm{m} / \mathrm{m})$.

Cet indice est calculé en utilisant l'extension Spatial Analyst d'ArcGis, et repose sur un calcul de l'écoulement suivant le modèle « multiple flow D-Infinity» (Tarboton, 1997) implémenté dans « Taudem $5.1 »$ (Tarboton, 2013). L'indice calcule la probabilité qu'une particule d'un point donné arrive à la cible finale et est compris entre $[-\infty,+\infty]$ (fig. 4). Dans cette étude, qui a pour but d'étudier le couplage versant/lit à la lumière de la crue de juin 2013, le réseau hydrographique principal a été choisi comme cible finale de la particule. Ce masque du réseau hydrographique principal a été créé avec l'outil ArcHydro d'ArcGis, avec un seuil de $4 \mathrm{~km}^{2}$. Ce seuil a été choisi dans un objectif de comparaison interbassins. Il correspond, d'après nos observations, au seuil à partir duquel la crue a été morphogène sur le réseau hydrographique. Le MNT utilisé présente une résolution spatiale de $5 \mathrm{~m}$ et une précision verticale métrique. D'après Crema et Cavalli (2018), l'estimation de la connectivité sédimentaire avec l'IC peut être appliquée à des Modèles Numériques de Terrain de résolution moyenne compris entre 5 et $30 \mathrm{~m}$.

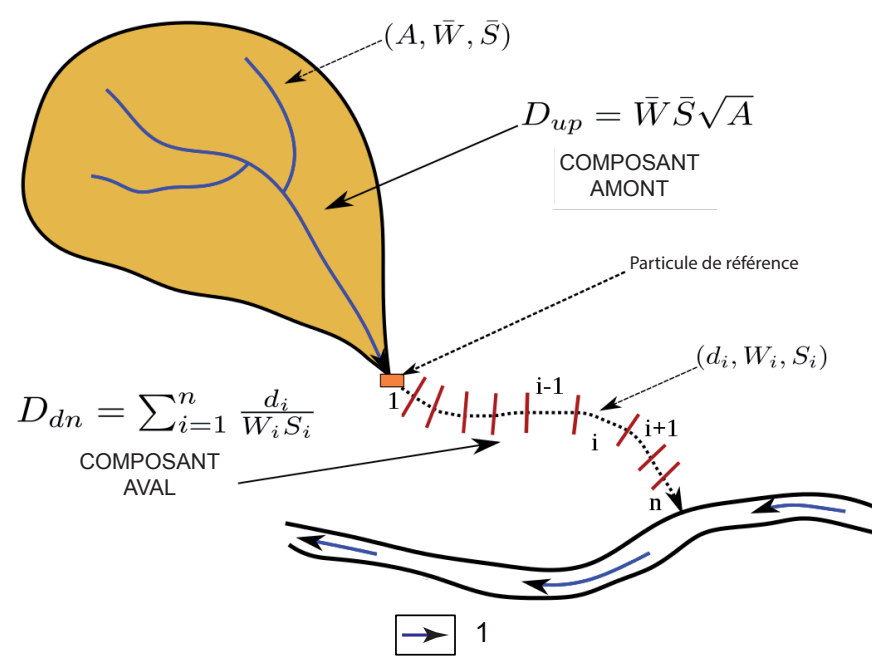

Fig. 4 - Schéma de l'Indice de connectivité sédimentaire, d'après Borselli et al. (2008), traduit à partir de Cavalli et al. (2013).

1. Réseau hydrographique principal ou zone de stockage locale (e.g., rivière, route, lac, zone urbaine).

Fig. 4 - Diagram of Index of connectivity, from Borselli et al. (2008), translate from Cavalli et al. (2013).

1. Main river system or local storage area (e.g., river, road, lake, urban area). 

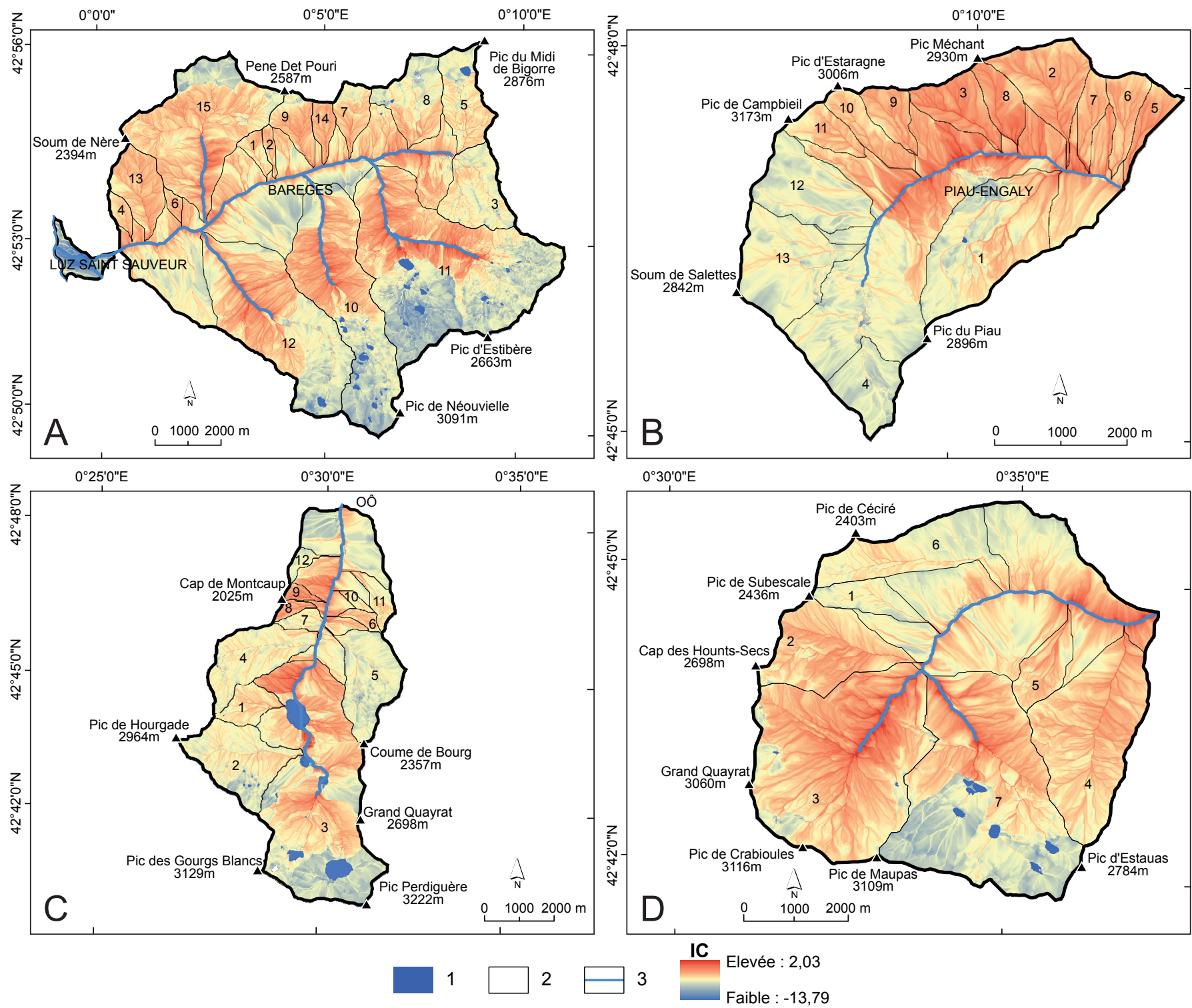

Fig. 5 - Cartographie de l'indice de connectivité sédimentaire au réseau hydrographique principal des quatre bassins versants à partir du MNT $5 \mathrm{~m} \odot$ IGN.

1. Lacs ; 2. Sous-bassins versants ; 3. Réseau hydrographique principal. A : Bassin du Bastan ; B : Bassin de la Neste de Badet ; C : Bassin de la Neste d'Oô ; D : Bassin du Lys.

Fig. 5 - Map of the Index of connectivity to the main channels for the 4 catchments from 5-m DTM $\odot$ IGN.

1. Lakes; 2. Subcatchments; 3. Main channels. A: Bastan basin; B: Neste de Badet basin C: Neste d' Oô basin; D: Lys basin.

\section{Résultats}

4.1. Les connectivités sédimentaires structurelle et fonctionnelle des bassins versants et sous-bassins versants

La distribution de la connectivité sédimentaire des quatre bassins et de leurs sous-systèmes associés est illustrée sur la Figure 5. Les valeurs se répartissent de 2,03 à -13,79. La Figure 6 représente les statistiques principales de la connectivité, couplées avec la taille des sous-bassins versants, la dynamique à l'exutoire et la contribution en surface de zones actives lors de la crue de juin 2013. Deux variables retiennent l'attention : les valeurs moyennes et maximales de connectivité.

Les zones de gorges du bassin du Lys et dela Neste d'Oô présentent les valeurs les plus élevées. Les sous-bassins versants à forte connectivité moyenne sont, d'un point de vue topographique, assez homogènes, à forte pente et favorables au déclenchement

de processus de versant. Le processus avalancheux a davantage d'importance que celui des laves torrentielles. Les sous-bassins $\mathrm{n}^{\circ} 3$ et $\mathrm{n}^{\circ} 8$ du bassin de la Neste de Badet ont une moyenne de valeur de connectivité supérieure à -4 .

À contrario, la connectivité moyenne est plus faible dans les grands sous-bassins versants aux têtes de bassins plus élevées pour lesquels la dynamique est majoritairement torrentielle et où le processus de transport dominant à l'exutoire est le charriage. Ils affichent une connectivité très hétérogène, avec les valeurs les plus faibles là où les parties amont présentent une morphologie en cirques et lacs glaciaires et des fonds de vallée plus larges ralentissant ou annihilant le transfert sédimentaire. Mais ils présentent également les valeurs maximales les plus élevées, dans les zones situées en fond de vallée à proximité du réseau hydrographique principal. Par exemple, les sous-bassins $n^{\circ} 10$ du bassin du Bastan (torrent de la Glère) et $\mathrm{n}^{\circ} 3$ du bassin de la Neste d'Oô (Neste d'Oô amont) présentent une valeur maximale 

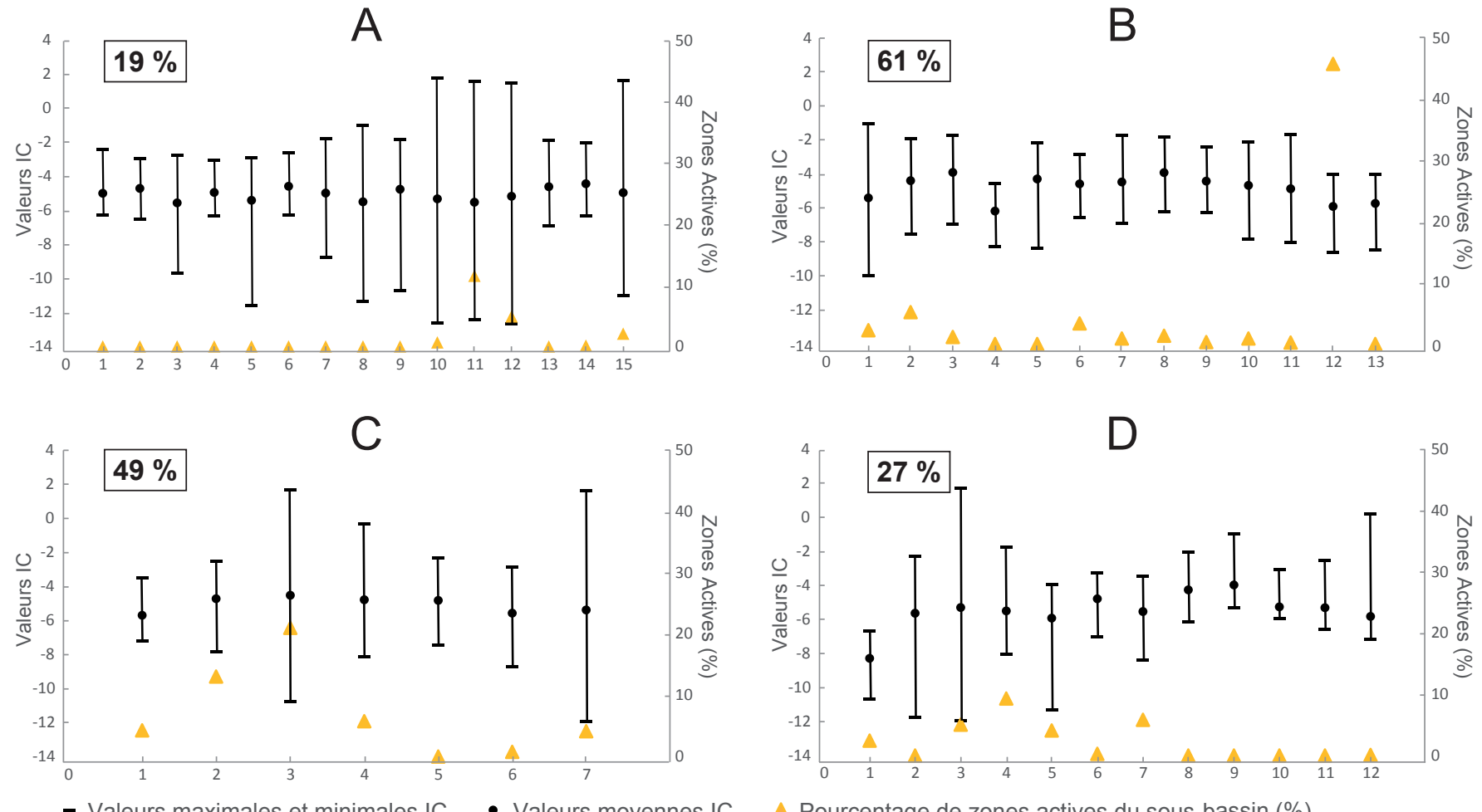

- Valeurs maximales et minimales IC - Valeurs moyennes IC

$\Delta$ Pourcentage de zones actives du sous-bassin (\%)

$27 \%$ Contribution des sous-bassins à la somme des zones actives du bassin

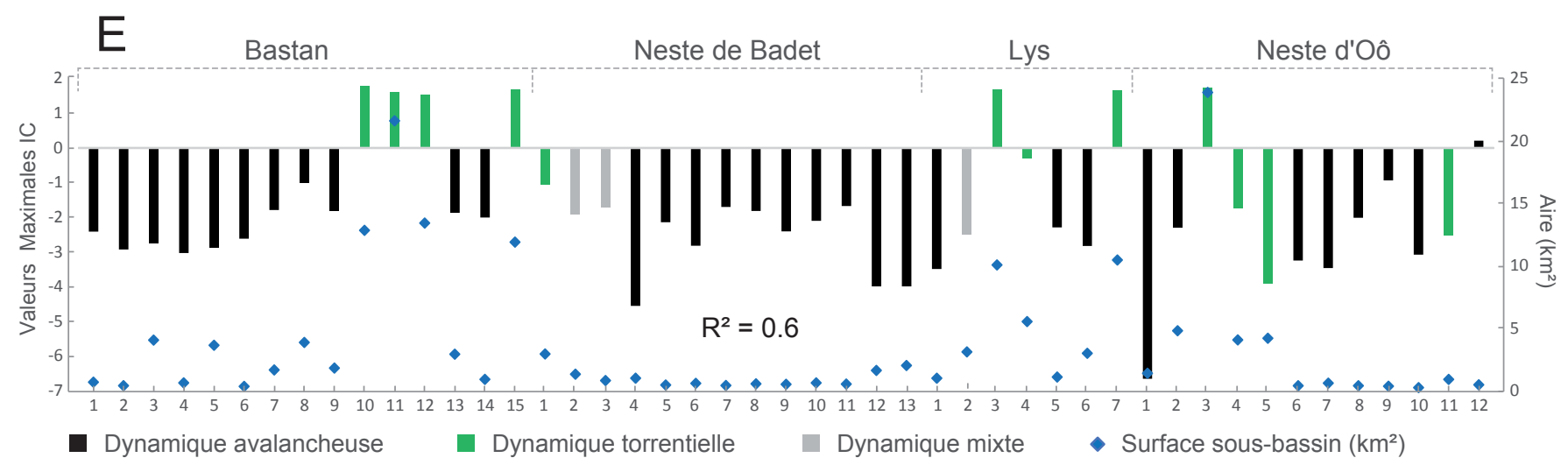

Fig. 6 - Statistiques principales de la connectivité sédimentaire des sousbassins versants.

A : Sous-bassins du Bastan ; B : Sous-bassins de la Neste de Badet ; C : Sous-bassins du Lys ; D : Sous-bassins de la Neste d'Oô; E : Valeurs maximales de l'IC par sous-bassins.

supérieure à 1,7. Ce rapport entre la surface du sous-bassin et les valeurs maximales est associé à un coefficient de détermination de 0,6 qui détermine une certaine corrélation. Cette corrélation s'explique en partie par le masque du réseau hydrographique principal cible. En effet, le réseau hydrographique cible possède un bassin de réception d'au moins $4 \mathrm{~km}^{2}$ favorisant les valeurs de forte connectivité sur le réseau hydrographique des grands bassins. Cependant, les valeurs maximales sont intéressantes à analyser car d'après Recking (2012), le transport sédimentaire et la recharge d'un système peuvent être importants lorsque le torrent est connecté à une source active.

$\mathrm{Au}$ même titre que les valeurs maximales, la répartition des surfaces contributives des sous-bassins versants lors de la crue de juin 2013 (fig. 6) fait ressortir l'importance des bassins de grande taille par rapport aux petits bassins. Les tributaires de
Fig. 6 - Main statistics of the sedimentary connectivity of the subcatchments.

A: Bastan subcatchments; B: Neste de Badet subcatchments; C: Lys subcatchments; D: Neste d'Oô subcatchments; E: IC Maximum values per subcatchments.

rive gauche du Bastan ( $\mathrm{n}^{\circ} 11$ et 12 ), le $\mathrm{n}^{\circ} 12$ du bassin de la Neste de Badet, la Neste d'Oô amont $\left(\mathrm{n}^{\circ} 3\right)$, le Lys amont $\left(\mathrm{n}^{\circ} 3\right)$ sont les principaux contributeurs de la crue de juin 2013 dans leurs bassins respectifs. Cependant, leur part dans la contribution à la totalité du bassin reste faible pour le Bastan et la Neste d'Oô, avec respectivement $19 \%$ et $27 \%$ (fig. 6). En effet, la majeure partie des surfaces contributives sur ces deux bassins (81\% pour le Bastan et $73 \%$ pour la Neste d'Oô) n'est pas issue des tributaires mais du fond de vallée à proximité du collecteur principal. À l'inverse, les sous-bassins tributaires de la Neste de Badet ont fourni $61 \%$ des zones actives lors de la crue. Quant à l'analyse de l'indice de compacité de Gravelius, elle ne permet pas de dégager une tendance de l'influence de la forme des sousbassins versants sur la connectivité sédimentaire, et elle n'est donc pas présentée ici. 


\subsection{Les connectivités sédimentaires structurelle et fonctionnelle des formations superficielles}

Le couplage versant/lit cible différentes formes de stockage sédimentaire en lien direct, indirect ou intermittent avec le chenal (Harvey, 2001). La cartographie géomorphologique des formations superficielles permet de dresser un premier état des lieux du couplage sédimentaire (Schlunegger et al., 2009 ; Theler, 2010). Sur les quatre bassins étudiés, $64 \%$ des formations superficielles sont des dépôts glaciaires hérités (fig. 4) et ils représentent $36 \%$ de la superficie totale. Les moraines dénudées $(17 \%)$ et les dépôts glaciaires/talus d'éboulis indifférenciés (11\%) constituent les deux autres types de formations majoritairement présents dans ces bassins. Ces trois types de formations d'origine majoritairement glaciaire représentent au total $90 \%$ des formations superficielles. Les moraines dénudées, situées dans les cirques glaciaires, sont les dépôts les moins connectés avec une valeur moyenne de $-5,88$, et sont les plus éloignés du réseau hydrographique (fig. 7).

Les autres formations post-glaciaires, situées en fond de vallée, comme les cônes de déjection mixtes et les dépôts fluvio-torrentiels, représentent à peine plus de $3 \%$ des formations superficielles mais sont les plus connectés au réseau hydrographique principal, avec une valeur moyenne de $-4,36$. Pour les cônes de déjection torrentiels, la faible valeur moyenne de connectivité doit cependant être nuancée. En effet l'analyse inclut le cône du Bastan à sa confluence avec le gave de Pau à Luz-Saint-Sauveur qui contribue à réduire fortement la moyenne de l'indice de connectivité. En effet, ce cône à pente très faible est un des cônes torrentiels les plus vastes des Pyrénées. Une partie du cône a été active lors de la crue de 2013 comme l'atteste l'élargissement de la bande active et l'érosion des dépôts adjacents, mais sa faible pente minore sa connexion dans le calcul de l'IC. L'analyse structurelle de la connectivité sédimentaire des formations superficielles indique donc une tendance à une cascade sédimentaire théorique très courte entre les bas de versant et les chenaux principaux. La déconnexion des formations superficielles de versant et des stocks paraglaciaires amont est peu propice à l'efficacité de la connectivité sédimentaire.

En ce qui concerne les zones actives, les dépôts fluvio-torrentiels, bien que faiblement représentés, concourent à plus de $46 \%$ des surfaces actives lors de la crue (fig. 7). Ces unités comprennent les matériaux remaniés dans le chenal mais également les érosions en lit majeur (fig. 8). Les dépôts glaciaires/talus d'éboulis indifférenciés représentent quant à eux $23 \%$ des zones actives, et se rencontrent majoritairement dans le bassin de la Neste de Badet. Issus de parties intermédiaires du bassin versant et des pieds de versant, ils ont été remobilisés et acheminés au chenal par charriage hyperconcentré et lave torrentielle. Par ailleurs, l'analyse par photo-interprétation a permis de déceler l'absence de laves torrentielles dans les bassins de la Neste d'Oô, du Lys et du Bastan lors de la crue. La présence de neige dans les parties les plus élevées au moment de la crue, ainsi que la correction des ravines historiquement actives au milieu du $\mathrm{XIX}^{\mathrm{e}}$ siècle jusqu'au début du $\mathrm{XX}^{\mathrm{e}}$ siècle, expliquent en partie la
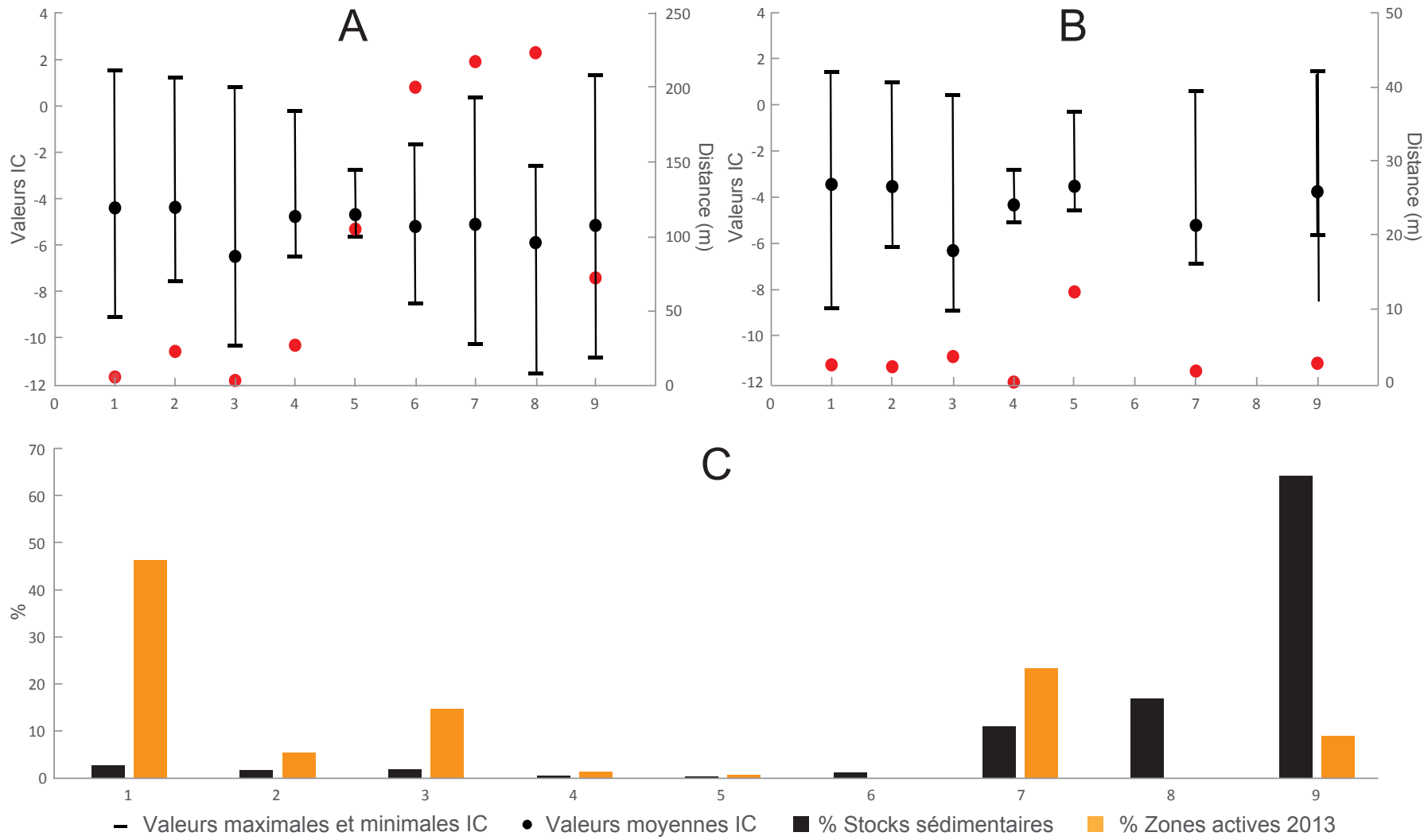

- Moyenne des plus faibles distances au réseau hydrograpique (BD Carthage IGN)

Fig. 7 - Statistiques principales de la connectivité des formations superficielles.

A : IC des stocks sédimentaires ; B : IC des zones actives de la crue de juin 2013 ; $\mathrm{C}$ : Comparaison de la répartition des types de formations superficielles et des zones actives. 1. Dépôts fluvio-torrentiels ; 2. Cônes de déjection mixtes ; 3. Cônes de déjection torrentiels ; 4. Ravines ; 5. Glissements de terrain ; 6. Éboulis ; 7. Dépôts glaciaires/Talus d'éboulis indifférenciés; 8 . Moraines; 9. Dépôts glaciaires hérités.
Fig. 7 - Main statistics of the connectivity of surficial geology.

A: IC of surficial sediment storages; B: IC of the active zones; C: Comparison of the distribution of surficial sediment storages and active zones. 1. Alluvial deposits; 2. Mixed debris cones; 3. Debris cones; 4. Gullies; 5 . Landslides; 6. Talus slope; 7. Undistinguished glacial deposits/talus slope; 8. Moraine deposits; 9. Inherited glacial deposits. 

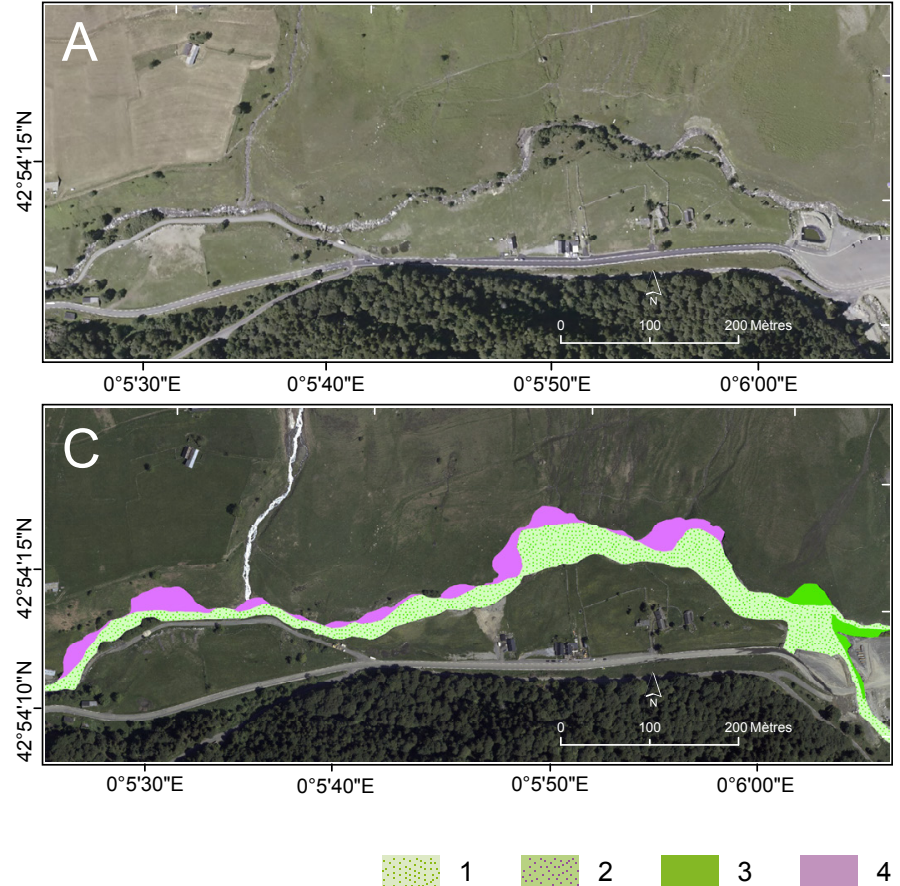

2

3

Fig. 8 - Exemple de la plaine alluviale de Tournaboup.

Types de stockage sédimentaire : 1. Dépôts fluvio-torrentiels ; 2 . Cônes de déjection mixtes ; 3. Cônes de déjection torrentiels ; 4. Dépôts glaciaires hérités ; 5. Zones actives ; 6. Réseau hydrographique principal. A : Orthophotographie IGN de 2010 ; B : Photographie aérienne RTM de juillet 2013 ; C : Zones actives de la crue de juin 2013 ; D : Indice de connectivité et zones actives de juin 2013.

contribution sédimentaire limitée des têtes de bassin versant. Elle s'explique également par la répartition et la connectivité des formations superficielles des zones amont. Les formations superficielles dénudées de tête de bassin comme les moraines et les éboulis apparaissent comme des dépôts non contributifs à la constitution de la charge solide de la crue de juin 2013. À contrario, ce sont les formations de fond de vallée et de pied de versant qui ont largement été remaniées (fig. 8).

Les formations superficielles actives ont des valeurs de connectivité supérieure d'en moyenne 1 point par rapport aux formations de l'inventaire (fig. 7). Les dépôts fluvio-torrentiels et les cônes mixtes actifs possèdent la connectivité la plus forte, avec des valeurs moyennes proches de $-3,5$. La différence la plus marquante se situe entre les dépôts glaciaires hérités et les dépôts glaciaires hérités actifs allant de $-5,14$ à $-3,76$.

\section{Discussion}

En accord avec les observations de terrain, la modélisation de l'indice de connectivité montre une faible connectivité sédimentaire des têtes de bassin versant et des formations superficielles du domaine glaciaire et périglaciaire actuel. Les cirques glaciaires granitiques concentrent des dépôts de type morainique et des éboulis. Le profil en long de ces vallées glaciaires amont, voyant alterner verrous et lacs d'ombilics, est un obstacle à la connectivité longitudinale et affecte la connectivité versant/lit (Brardinoni et Hassan, 2006; Kuo et Brierley, 2013 ; Hoffmann et al., 2013). Lors de la crue de juin 2013, ces dépôts n’ont pas participé à la constitution de la charge solide acheminée vers le réseau hydrographique.

Dans ces quatre bassins où la crue a été la plus morphogène, la Neste de Badet fait office d'exception au vu de la dynamique des trois autres bassins versants. Sa morphologie est différente, avec des
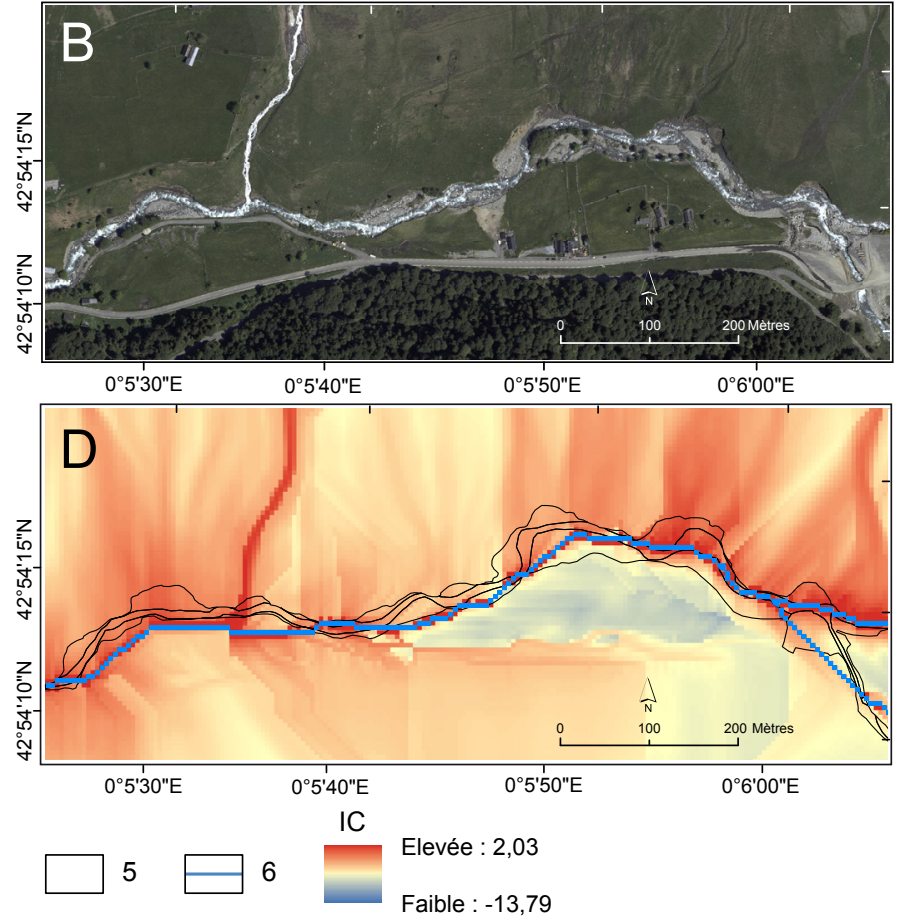

Fig. 8 - Example of the alluvial plain of Tournaboup.

Sediment storage types: 1. Alluvial deposits; 2. Mixed debris cones; 3.Debris cones; 4. Inherited glacial deposits; 5. Actives areas; 6 . Main channels. A: Orthophotography IGN 2010; B: Aerial photography RTM in July 2013; C: Active zones of June 2013 flood; D: Connectivity Index and active zones of June 2013 flood.

versants plus raides, un fond de vallée plus étroit et une absence de cirques glaciaires. Ses sous-bassins versants possèdent une connectivité structurelle et fonctionnelle plus importante. Lors de la crue, la Neste de Badet a été alimentée à plus de $60 \%$ par des surfaces actives sur les tributaires. Les $40 \%$ restant proviennent de dépôts glaciaires et post-glaciaires du fond de vallée du collecteur principal. La cascade sédimentaire semble être efficace avec un remaniement des dépôts de versant par les processus avalancheux et torrentiels vers le chenal.

Dans les trois autres bassins, la crue a mis en lumière une mobilisation des formations superficielles des fonds de vallées du collecteur principal et des sous-systèmes de grande taille à processus de charriage dominant. La modélisation morphométrique met en évidence la remobilisation du matériel présent dans le chenal d'une part, et des formations superficielles adjacentes d'autre part via l'analyse des valeurs maximales de l'IC. La connectivité moyenne structurelle est plus forte dans les petits sous-bassins homogènes à forte pente comme par exemple des sous-bassins de rive droite $\mathrm{du}$ Bastan. Ce sont pour certains des bassins historiquement producteurs d'avalanches, corrigés depuis la fin du XIX ${ }^{e}$ siècle et qui ont été inactifs pendant la crue.

Le croisement entre l'analyse des sous-bassins versants, des formations superficielles et des zones actives selon l'IC (indice de connectivité) met en exergue l'importance de certains facteurs de contrôle de la connectivité. Si la forme des bassins et sous-bassins ne semble pas jouer de rôle déterminant, la taille ainsi que la répartition des formations superficielles sont des variables qui influencent significativement la connectivité sédimentaire dans les Pyrénées centrales. En effet, l'absence de formations superficielles connectées dans le domaine glaciaire et périglaciaire actuel est remarquable sur les trois bassins. La part la plus importante de la contribution des formations superficielles concerne les dépôts de fond de vallée du 
collecteur principal. Logiquement, les dépôts fluvio-torrentiels sont les formations les plus contributives et les plus connectées au réseau hydrographique principal. En crue, il est généralement admis que le chenal est la zone principale de fourniture sédimentaire (Bardou, 2002) avec les érosions de berges (Harvey, 2001). Dans notre étude, l'élargissement des bandes actives a remanié essentiellement des dépôts fluvio-torrentiels présents dans les zones de divagation et des dépôts glaciaires/talus d'éboulis indifférenciés adjacents au chenal.

Dans les Alpes italiennes et suisses, Cavalli et al. (2016) et Messenzehl et al. (2014) notent, à travers l'analyse des différents stocks sédimentaires et des topo-séquences, une plus forte connectivité des formations superficielles localisées dans les sections aval et intermédiaires des vallées proches de l'exutoire. En effet, avec des têtes de bassins déconnectées, les formations superficielles de bas de versant, dans les sections aval et intermédiaires des vallées, sont les plus connectées au réseau hydrographique. Par ailleurs, ces deux études montrent des valeurs de connectivité supérieures aux quatre bassins des Pyrénées centrales. La connectivité sédimentaire au réseau hydrographique des bassins torrentiels étudiés dans les Alpes (entre 6 et $8,5 \mathrm{~km}^{2}$ ) est comprise entre $-2,35$ et $-2,6$ alors qu'elle est comprise entre $-3,8$ et $-6,2$ dans les sous-systèmes des quatre bassins pyrénéens (entre 0,25 et $21 \mathrm{~km}^{2}$ ) pour une moyenne supérieure à -5 . Globalement la connectivité sédimentaire sur les quatre bassins étudiés est relativement faible. Ces résultats sont cependant à nuancer en fonction de la résolution des MNT utilisés, à savoir 1 et $2 \mathrm{~m}$ pour Cavalli et al. (2016) et Messenzehl et al. (2014) contre $5 \mathrm{~m}$ dans notre étude, et du réseau hydrographique cible. Les fonds de vallées étroits avec des versants raides sont décrits par Fryirs (2005) comme étant des caractéristiques influençant l'efficacité de la connectivité sédimentaire. Le bassin de la Neste de Badet possède ces caractéristiques morphométriques sans cirques glaciaires amont étendus et avec des formations superficielles paraglaciaires actives le long des versants. Ce bassin possède le couplage versant/lit le plus efficace de notre étude et il est le plus connecté selon l'IC. Dans les trois autres bassins, l'efficacité ou même le (dys)fonctionnement de la cascade sédimentaire pose question. La connectivité est efficace uniquement entre le chenal et les formations adjacentes principalement par érosion de berge ou sapement de pied de versant.

À titre de comparaison, dans le Queyras (bassin-versant du Guil) où les apports sédimentaires sont importants lors des crues ou laves torrentielles (Arnaud-Fassetta et Fort, 2004 ; Graff et al., 2018), une partie des stocks sédimentaires hérités de la dernière glaciation et des réajustements post-glaciaires n'est plus connectée au système géomorphologique actuel (Carlier et al., 2018). Cependant, la cascade sédimentaire s'active lors d'évènements Basse Fréquence Haute Magnitude (BFHM), en partant des versants par coulées de débris et des tributaires par laves torrentielles jusqu'au collecteur principal du Guil (Arnaud-Fassetta et Fort, 2004). Elle est aussi plus fréquemment efficace lors d'évènements de plus faible magnitude, uniquement sur les drains élémentaires (Graff et al., 2018) et sous bassins-versants. Dans les Pyrénées centrales, la cascade sédimentaire semble rester très courte même en situation de crue BFHM et peu efficace pendant les temps de récupération entre deux crises hydro-géomorphologiques. La connectivité sédimentaire est influencée, comme l'a montré Bracken et al. (2015), par la magnitude et la fréquence des crues. L'influence du retrait des glaciers a été par exemple avancée comme autre cause de la variabilité temporelle de la connectivité sédimentaire (Cavalli et al., 2016; Goldin et al., 2016 ; Micheletti and Lane, 2016). Au vu de leur faible emprise, du stade avancé de leur recul (René, 2011) et de leur large déconnexion, il est fort probable que le désenglacement des Pyrénées centrales n'influencera en rien la connectivité sédimentaire future. Cependant, il est avéré que la connectivité longitudinale et latérale est moins forte aujourd'hui qu'elle ne l'a été en période post Petit Âge Glaciaire (1850-1940), avec une récurrence d'évènements torrentiels et avalancheux majeurs. L'IC n'intègre pas le rôle, la fréquence et la magnitude des évènements et processus extrêmes, de même qu'il n'inclut pas le rôle de la végétation. Mais l'analyse de la connectivité fonctionnelle des zones actives lors d'une crue majeure valide la complémentarité du croisement entre les formations superficielles et la modélisation morphométrique. La crue permet d’observer une connectivité exacerbée à un instant donné. Dans la mesure où les valeurs de connectivité des zones actives sont supérieures à celle des stocks sédimentaires hérités, l'étude confirme le potentiel de la modélisation de la connectivité à l'aide du SIG dans l'estimation de la connectivité sédimentaire, dans la caractérisation du rôle des formations superficielles lors d'une crue, et plus globalement dans la compréhension de la dynamique sédimentaire à l'échelle du bassin versant (Crema et Cavalli, 2018 ; Cavalli et al., 2016 ; Tiranti et al., 2016). Ce modèle est efficace pour examiner l'influence des paramètres de contrôle de la connectivité sédimentaire, e.g., la taille des bassins versants pour le cas de la haute chaîne pyrénéenne. Il permet également d'analyser la susceptibilité à la contribution des différentes formations superficielles en fonction de leur type et de leur localisation dans le bassin.

\section{Conclusion}

Cette étude se base sur l'utilisation de l'indice morphométrique de la connectivité sédimentaire proposé par Cavalli et al. (2013). Son croisement avec la cartographie géomorphologique des formations superficielles a permis la caractérisation de la connectivité sédimentaire dans la haute chaine pyrénéenne. Les petits sousbassins versants sont les plus connectés (valeurs moyennes) mais n'ont pas été contributifs lors de la crue. Alors que les sous-bassins versants de plus grande taille possèdent une connectivité moyenne moindre mais une contribution supérieure. La connectivité sédimentaire est ici contrôlée par la taille des sous-bassins versants mais aussi par la distribution des formations superficielles. Les dépôts glaciaires ou post-glaciaires situés dans les cirques glaciaires déconnectés ne participent pas à la constitution de la charge solide du réseau hydrographique. En revanche, les dépôts post-glaciaires et certains dépôts glaciaires hérités, adjacents au collecteur principal du bassin ou des sous-bassins de grande taille, sont les plus connectés et les plus contributifs. De fait, la cascade sédimentaire efficace semble être très courte dans les Pyrénées centrales et mérite d'être approfondie dans l'optique d'une comparaison avec d'autres massifs montagneux. La crue majeure de juin 2013 a permis d'observer une connectivité exacerbée des formations superficielles avec le réseau hydrographique à un instant donné. La comparaison entre la connectivité structurelle et fonctionnelle de la crue valide la fiabilité de l'indice d'une part et, d'autre part, montre la vulnérabilité des formations superficielles allochtones et subautochtones de fond de vallée lors d'évènement Basse Fréquence Haute Magnitude.

\section{Remerciements}

Cette étude a été réalisée dans le cadre du programme interdisciplinaire DYNHAPYR, financé par l'ancienne région Midi-Pyrénées (aujourd'hui Occitanie) et l'Université Toulouse Jean Jaurès. Elle fait partie d'un travail plus large mené dans le cadre d'une thèse de l'Université Toulouse 2 Jean Jaurès sur la dynamique torrentielle dans les Pyrénées centrales. 


\section{Références}

Arnaud-Fassetta G., Fort M. (2004) - La part respective des facteurs hydroclimatiques et anthropiques dans l'évolution récente (1956-2000) de la bande active du Haut-Guil, Queyras, Alpes françaises du Sud. Méditerranée, 102 (1-2), 143-156. DOI : $10.3406 /$ medit.2004.3350

Bardou E. (2002) - Méthodologie de diagnostic des laves torrentielles sur un bassin versant alpin. PhD Thesis, École Polytechnique Fédérale de Lausanne, Lausanne, 171 p.

Borselli L., Cassi P., Torri D. (2008) - Prolegomena to sediment and flow connectivity in the landscape: a GIS and field numerical assessment. Catena, 75, 268-277.

DOI : 10.1016/j.catena.2008.07.006

Bracken L.J., Turnbull L., Wainwright J., Bogaart P. (2015) Sediment connectivity: a framework for understanding sediment transfer at multiple scales. Earth Surface Processes and Landforms 40, 177-188.

DOI : $10.1002 /$ esp.3635

Brardinoni F., Hassan M.A. (2006) - Glacial erosion, evolution of river long profiles, and the organization of process domains in mountain drainage basins of coastal British Columbia. Journal of Geophysical Research, 111, F01013.

DOI : 10.1029/2005JF000358

Bravard J.-P., Petit F. (2000) - Les cours d'eau. Dynamique du système fluvial. Paris, Colin, 2e édition, $222 \mathrm{p}$.

Brierley G.J., Fryirs K.A., Jain V. (2006) - Landscape connectivity: The geographic basis of geomorphic applications. Area, 38 (2), 165-174.

DOI : $10.1111 /$ j.1475-4762.2006.00671.x

Caine N., Swanson F.J. (1989) - Geomorphic coupling of hillslope and channel systems in two small mountain basins. Zeitschrift fur Geomorphologie, 33, 189-203.

Campagne A. (1902) - La Vallée de Barèges et le reboisement : les torrents - le désastre de 1897 : Les avalanches, Ulan Press, 167p.

Carlier B., Carlier G., Gance J., Provost F., Lissak C., Graff K., Viel V., Arnaud-Fassetta G., Fort M., Bétard F., Madelin M., Malet J.P., Cossart E. (2018) - Distribution spatiale et estimation des volumes sédimentaires dans un bassin-versant de schistes lustrés : l'exemple du Peynin, Queyras, Alpes du Sud. Géomorphologie : Relief, Processus, Environnement, 24 (1), 59-76.

DOI : 10.4000/geomorphologie.11994

Cavalli M., Tarolli P., Dalla Fontana G., Marchi L. (2016) Multi-temporal analysis of sediment source areas and sediment connectivity in the Rio Cordon catchment (Dolomites). Rendiconti Online Della Societa Geologica Italiana, 39, 27-30. DOI : $10.3301 /$ ROL.2016.39

Cavalli M., Trevisani S., Comiti F., Marchi L. (2013) Geomorphometric assessment of spatial sediment connectivity in small Alpine catchments. Geomorphology, 188, 31-41. DOI : 10.1016/j.geomorph.2012.05.007

CHE-Confederación Hidrográfica del Ebro (2014) - Informe de la avenida del 17 al 20 de junio de 2013 en la cuenca del río Garona, Ministerio de Agricultura, Alimentación y Medio Ambiente, Gobierno de España, 73 p.

Chiverrell R.C., Foster G.C., Marshall P., Harvey A.M., Thomas G.S.P. (2009) - Coupling relationships: Hillslopefluvial linkages in the Hodder catchment, NW England. Geomorphology 109, 222-235.

DOI : 10.1016/j.geomorph.2009.03.004

Chorley R.J., Schumm S.A., Sudgen S.E. (1984) - Geomorphology. Routledge, London, New York, 607 p.
DOI : $10.1017 / S 0016756800035573$

Cossart E. (2016) - L'(in)efficacité géomorphologique des cascades sédimentaires en question : les apports d'une analyse réseau, Cybergeo: European Journal of Geography, document 778.

DOI : $10.4000 /$ cybergeo. 27625

Cossart E., Fressard M. (2017) - Assessment of structural sediment connectivity within catchments: insights from graph theory. Earth Surface Processes and Landforms, 5, 253-268. DOI : $10.5194 /$ esurf-5-253-2017

Cossart E., Lissak C., Viel V. (2017) - La géomorphologie des bassins-versants sous l'angle de la connectivité : est-ce réinventer la roue ou changer de paradigme ?, Géomorphologie : Relief, Processus, Environnement, 23 (4), 281-287.

DOI : $10.4000 /$ geomorphologie. 11893

Crema S., Cavalli M. (2018) - SedInConnect: A stand-alone, free and open source tool for the assessment of sediment connectivity. Computers Geosciences, 111, 39-45.

DOI : 10.1016/j.cageo.2017.10.009

Croke J., Mockler S., Fogarty P., Takken I. (2005) - Sediment concentration changes in runoff pathways from a forest road network and the resultant spatial pattern of catchment connectivity. Geomorphology, 68, 257-268.

DOI : 10.1016/j.geomorph.2004.11.020

Dewolf Y., Bourrié G. (2008) - Les formations superficielles : genèse, typologie, classification, paysages et environnements, ressources et risques. Ellipses, 829 p.

ISBN : 978-2-7298-3556-9

Eaton L.S., Morgan B.A., Kochel R.C., Howard A.D. (2003) Quaternary deposits and landscape evolution of the central Blue Ridge of Virginia. Geomorphology, 56, 139-154.

DOI : $10.1016 /$ S0169-555X(03)00075-8

ETRM-Eaux Torrents et Rivières de Montagne (2014) - Crue de juin 2013 sur le bassin versant de la Pique en amont de Bagnères-de- Luchon : Analyse du transport solide. Direction Départementale des territoires de la Haute Garonne, 46 p.

Evans I.S. (2012) - Geomorphometry and landformmapping: What is a landform ? Geomorphology 137, 94-106.

DOI : 10.1016/j.geomorph.2010.09.029

Fryirs K. (2005) - Linking landscape processes and river systems: Assessing implications of catchment-scale (dis)connectivity of sediment movement on river sensitivity, recovery and river management. In Rutherfurd, I.D., Wiszniewski I., Askey-Doran M.J., Glazik, R. (Eds.) Proceedings of the $4^{\text {th }}$ Australian Stream Management Conference: Linking Rivers to Landscapes. Department of Primary Industries, Water and Environment, Hobart, Tasmania. 256-259.

Fryirs K. (2013) - (Dis)Connectivity in catchment sediment cascades: a fresh look at the sediment delivery problem. Earth Surface Processes and Landforms, 38, 30-46.

DOI : 10.1002 /esp. 3242

Fryirs K., Brierley G.J. (2001) - Variability in sediment delivery and storage along river courses in Bega catchment, NSW, Australia: implications for geomorphic river recovery. Geomorphology, 38, 237-265.

DOI : 10.1016/S0169-555X(00)00093-3

Fryirs K.A., Brierley G.J., Preston N.J., Kasai M. (2007) - Buffers, barriers and blankets: the (dis)connectivity of catchment-scale sediment cascades. Catena, 70, 49-67. DOI : $10.1016 /$ j.catena.2006.07.007

Gay A., Cerdan O., Mardhel V., Desmet M. (2015) - Application of an index of sediment connectivity in a lowland area. Journal of Soils Sediments, 3, 1-14.

DOI : $10.1007 / \mathrm{s} 11368-015-1235-y$ 
Goldin B., Rudaz B., Bardou E. (2016) - Application of a sediment connectivity GIS-based index in a basin undergoing glacier retreat: the case study of the Navizence catchment. Rendiconti Online Della Societa Geologica Italiana, 39, 35-38.

DOI : $10.3301 /$ ROL.2016.41

Graff K., Viel V., Carlier B., Lissak C., Madelin M., ArnaudFassetta G., Fort M. (2018) - Traçage sédimentaire d'une lave torrentielle dans le basin de Peyronnelle, Queyras, Alpes françaises du Sud. Géomorphologie : Relief, Processus, Environnement, 24 (1), 43-57.

DOI : 10.4000/geomorphologie.11967

Gran K.B., Czuba J.A. (2017) - Sediment pulse evolution and the role of network structure. Geomorphology, 277, 17-30.

DOI : 10.1016/j.geomorph.2015.12.015

Harvey A.M. (1991) - The influence of sediment supply on the channel morphology of upland streams: The Howgill Fells, northwest England. Earth Surface Processes and Landforms, 16, 675-684.

DOI : $10.1002 /$ esp.3290160711

Harvey A.M. (2001) - Coupling between hillslopes and channels in upland fluvial systems: implications for landscape sensitivity, illustrated from the Howgill Fells, northwest England. Catena, 42, 225-250.

DOI : $10.1016 / \mathrm{S} 0341-8162(00) 00139-9$

Harvey A.M. (2002) - Effective timescales of coupling within fluvial systems. Geomorphology, 44, 175-201.

DOI : $10.1016 /$ S0169-555X(01)00174-X

Harvey A.M. (2012) - The coupling status of alluvial fans and debris cones: a review and synthesis. Earth Surface Processes and Landforms, 37, 64-76.

DOI : $10.1002 /$ esp. 2213

Heckmann T., Schwanghart W. (2013) - Geomorphic coupling and sediment connectivity in an alpine catchment - exploring sediment cascades using graph theory. Geomorphology, 182, 89-103.

DOI : 10.1016/j.geomorph.2012.10.033.

Heckmann T., Schwanghart W., Phillips, J. D. (2015) Graph theory - Recent developments of its application in geomorphology. Geomorphology, 243, 130-146.

DOI : 10.1016/j.geomorph.2014.12.024

Hoffmann T., Müller T., Johnson E.A., Martin Y. (2013) Postglacial adjustment of steep, low-order drainage basins, Canadian Rocky Mountains. Journal of Geophysical Research: Earth Surface, 118, 2568-2584.

DOI : $10.1002 / 2013$ JF002846.

Hooke J.M. (2003) - Coarse sediment connectivity in river channel systems: a conceptual framework and methodology. Geomorphology, 56, 79-94.

DOI : $10.1016 /$ S0169-555X(03)00047-3

Hungr O., McDougall S., Bovis B. (2005) - Entrainment of material by debris flows. In Jakob M., Hungr O. (Eds.), Debris Flow Hazard and Related Phenomena. Springer, Heidelberg, Berlin, 135-158.

IGA-Inspection Générale de l'Administration., Conseil Général de l'Environnement et du Développement Durable., Conseil Général de l'Agriculture, de l'Alimentation et des Espaces Ruraux (2014) - Rapport de la mission d'appui et d'expertise sur les conséquences des intempéries ayant touché le Sud-Ouest et les Pyrénées les 18 et 19 juin 2013. 128p.

Kuo C.-W., Brierley G.J. (2013) - The influence of landscape configuration upon patterns of sediment storage in a highly connected river system. Geomorphology, 180-181, 255-266.

DOI : 10.1016/j.geomorph.2012.10.015
Liébault F. (2003) - Les rivières torrentielles des montagnes drômoises : évolution contemporainet fonctionnement géomorphologique actuel, massifs du Diois et des Baronnies, $\mathrm{PhD}$ thesis, Université Lumière Lyon 2, $358 \mathrm{p}$.

Lopez-Vicente M., Nadal-Romero E., Cammeraat E.L.H. (2016) - Hydrological connectivity does change over 70 Years of abandonment and afforestation in the Spanish Pyrenees. Land Degradation and Development, 28, 1298-1310.

DOI : $10.1002 / \mathrm{ldr} .2531$

Majesté-Menjoulàs C., Debon F., avec la collaboration de Driouch Y., Flachère H., Moreau H., Valéro J., Ternet Y. (1999) - Carte géol. France (1/50 000), feuille Gavarnie (1082). Orléans : BRGM. Notice explicative par C. Majesté-Menjoulàs, F Debon, P. Barrère avec la collaboration de T. Baudin, D. Leblanc, J. Astruc (1999), $158 \mathrm{p}$.

Merriam G. (1984) - Connectivity: a fundamental ecological characteristic of landscape pattern. In Brandt J., Agger P. (Eds), Methodological Landscape Ecology Research Planning proceedings, Roskilde University Centre, 5-15.

Messenzehl K., Hoffmann T., Dikau R. (2014) - Sediment connectivity in the high-alpine valley of Val Müschauns, Swiss National Park - linking geomorphic field mapping with geomorphometric modelling. Geomorphology, 221, 215-229. DOI : 10.1016/j.geomorph.2014.05.033

Micheletti N., Lane S.N. (2016) - Water yield and sediment export in small, partially glaciated Alpine watersheds in a warming climate. Water Resources Research, 52, 4924-4943.

DOI : 10.1002/2016WR018774

Nicoll T., Brierley G. (2016) - Within-catchment variability in landscape connectivity measures in the Garang catchment, Upper Yellow River. Geomorphology, 277, 197-209.

DOI : 10.1016/j.geomorph.2016.03.014

Pineda N., Prohom M., Serra A., Martí G., Garcia C., Velasco E., Gracia A. (2013) - Causes que van provocar la riuada a la Val d'Aran el 18 de juny de 2013. Jornada Gestión de las inundaciones, Barcelona, Spain, 120-125.

Recking A. (2012) - Influence of sediment supply on mountain streams bedload transport. Geomorphology, 175-176, 139-150. DOI : 10.1016/j.geomorph.2012.07.005

René P. (2011) - Régression des glaciers pyrénéens et transformation du paysage depuis le Petit Age Glaciaire. Sud-Ouest Européen, 32, 5-19. DOI : $10.4000 /$ soe. 639

Rice S.P. (2017) - Tributary connectivity, confluence aggradation and network biodiversity. Geomorphology, 27, 6-16.

DOI : 10.1016/j.geomorph.2016.03.027

Sandercock P.J., Hooke J.M. (2011) - Vegetation effects on sediment connectivity and processes in an ephemeral channel in SE Spain. Journal of Arid Environments, 75 (3), 239-254.

DOI : 10.1016/j.jaridenv.2010.10.005

Savi S., Schneuwly-Bollschweiler M., Bommer-Denns B., Stoffel M., Schlunegger F. (2013) - Geomorphic coupling between hillslopes and channels in the Swiss Alps. Earth Surface Processes and Landforms, 38, 959-969.

DOI : $10.1002 /$ esp. 3342

Schlunegger F., Badoux A., McArdell B.W., Gwerder C., Schnydrig D., Rieke-Zapp D., Molnar P. (2009) - Limits of sediment transfer in an alpine debris-flow catchment, Illgraben, Switzerland. Quaternary Science Reviews, 28, 1097-1105. DOI : 10.1016/j.quascirev.2008.10.025

Surian N., Righini M., Lucía A., Nardi L., Amponsah W., Benvenuti M., Borga M., Cavalli M., Comiti F., Marchi L., Rinaldi M., Viero A. (2016) - Channel response to extreme 
floods: insights on controlling factors from six mountain rivers in northern Apennines, Italy. Geomorphology, 272, 78-91.

DOI : 10.1016/j.geomorph.2016.02.002

Tarboton D.G. (1997) - A new method for the determination of flow directions and upslope areas in grid digital elevation models. Water Resources Research, 33, 309-319.

DOI : $10.1029 / 96$ WR03137

Tarboton D.G. (2013) - Taudem 5.1, Terrain Analysis Using Digital Elevation Models.[online] Available from:

http://hydrology.usu.edu/taudem/taudem5/

Theler D., Reynard E., Lambiel C., Bardou E. (2010) - The contribution of geomorphological mapping to sediment transfer evaluation in small alpine catchments. Geomorphology, 124, 113-123.

DOI : 10.1016/j.geomorph.2010.03.006

Tiranti D., Cavalli M., Crema S., Zerbato M., Graziadei M., Barbero S., Cremonini R., Silvestro C., Bodrato G., Tresso F.
(2016) - Semi-quantitative method for the assessment of debris supply from slopes to river in ungauged catchments. Science of the Total Environment, 554-555, 337-348.

DOI : $10.1016 /$ j.scitotenv.2016.02.150

Trevisani S., Cavalli M. (2016) - Topography-based flowdirectional roughness: potential and challenges. Earth Surface Dynamics, 4, 343-358.

DOI : $10.5194 /$ esurf-4-343-2016

Vigneau J.P. (1999) - Le fonctionnement climatique des Pyrénées, In Le dictionnaire des Pyrénées, Toulouse, Privat, 202-207.

Warburton J. (2010) - Sediment transfer in steep upland catchments (Northern England, UK): Landform and sediment source coupling, 165-184. In Otto J., Dikau R. (Eds.), Landform - Structure, evolution, process control: Proceeding of the International Symposium on Landform. Springer, Berlin, Heidelberg, $258 \mathrm{p}$.

DOI : 10.1007/978-3-540-75761-0_11

\section{Abridged English Version}

In mountainous areas, torrential basins and their associated tributaries are the site of increased sediment production and transport during a major flood. These high magnitude low frequency floods are periods of sediment connectivity activation between hillslopes and river channel and highlight its temporal and spatial variability.

There is a lack of study on both torrential dynamics and sediment connectivity in the Central Pyrenees. Furthermore the highmagnitude flood of June 2013 in the Central Pyrenees showed the role of inherited surficial sediment storages in the feeding of river sediment load. Compared to large historical floods, initial field observations led us to formulate the hypothesis of a low sediment delivery from torrential tributaries and headwaters. The study focused on the basins most affected during the flood namely the Bastan basin $\left(101 \mathrm{~km}^{2}\right)$, the Neste de Badet basin $\left(21 \mathrm{~km}^{2}\right)$, the Neste d'Oo basin $\left(43 \mathrm{~km}^{2}\right)$ and the Lys basin $\left(44 \mathrm{~km}^{2}\right)$ (fig. 1). These basins dominated by the granitic massif, the schisto-crystalline and schisto-calcareous metamorphic series. The purpose of this paper is to evaluate the role of inherited surficial sediment storages in the feeding of river sediment load and then to identify factors which explain sediment connectivity in order to characterize the sediment cascade of these Pyrenean basins. The sediment cascade represents a hierarchical nesting of sediment storage units (fig. 2). It allows a better evaluation of the coupling hillslope/channel by the study of the dynamic of sediment transfer. This work remobilizes the method proposed by Cavalli et al. (2013) and Messenzehl et al. (2014).

Sediment connectivity studied by distinguishing structural and functional connectivity. The analysis based on a GIS morphometric index of sediment connectivity (Cavalli et al., 2013) (fig. 4) derived from 5-m DTM. It modeled the potential connectivity between hillslopes and a target storage area. This mask created by ArcHydro tools with a threshold of $4 \mathrm{~km}^{2}$. In addition, classical geomorphic mapping of surficial sediment storages and active areas during the flood is carried out (fig. 3). On the other hand, the four basins cut in 47 sub-catchments by ArcHydro tools with a threshold of $0.2 \mathrm{~km}^{2}$. Geomorphic mapping, sub-catchments combined with the connectivity index (fig. 5) and allowed to compare the structural connectivity of sub-catchments and surficial sediment storages with functional connectivity during the flood (fig. 8).

Compare to the historical past major floods, it displayed the low sediment supply of small tributaries with high average connectivity (fig. 6). Headwater areas and glacial sediment storages didn't supply sediments during the flood and acted as disconnected areas according to the index. Moraines located in glacial cirques were the least connected deposits with an average of -5.88 and were the furthest from the main hydrographic network (fig. 7). Conversely, post glacial sediment storages in the valley bottom were the most connected and contributive stores. For example, torrential deposits represented $1.22 \%$ of all the deposits with a connectivity of -4.38 but contributed up to $46 \%$ of the active areas with an average IC of -3.44 . Lateral sediment connectivity occurred mainly between storages of the mid and low part of the valley which were adjacent to the main river. The share of the contribution of tributaries sub-catchments was low compared to the contribution of the main hydrographic network for the Bastan basin and Neste d'Oo basin (19\% and 27\%). The sediment cascade was effective only between the main collector and adjacent surficial sediment storages. Conversely, more than $60 \%$ of Neste de Badet basin supplied with active areas by tributaries. In this case, sediment cascade appears to be effective with reworking hillslopes deposits by avalanche and torrential processes to the channel.

Our study highlights the potential of the morphometric index supported by geomorphological mapping to characterize the central Pyrenean connectivity in a context of a low frequency high magnitude events. It showed factors which explain these dynamics such as the presence of deposits at the bottom valleys of major tributaries, the absence of connected sediment storages in the glacial and periglacial domain. Compared to other uses of the IC in the Alps (Cavalli et al., 2013; Messenzehl et al., 2014), sediment connectivity to the channel was relatively low. However, these results must be qualified according to the DTM resolution and the target hydrographic network. The activation of a relatively short sediment cascade during a low frequency high magnitudeflood showed the impact of the magnitude of a hydroclimatic event on sediment connectivity. The study underlines the spatial and temporal variability of sediment connectivity. 